\title{
Presença dos ácidos benzóico e sórbico em vinhos e sidras produzidos no Brasil
}

\author{
Presence of benzoic and sorbic acids in Brazilian wines and ciders
}

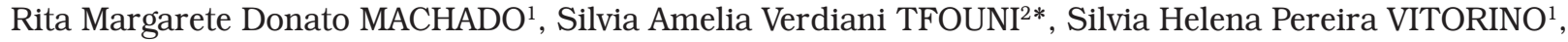 \\ Eduardo VICENTE ${ }^{2}$, Maria Cecília de Figueiredo TOLEDO ${ }^{1}$
}

\begin{abstract}
Resumo
O objetivo do presente trabalho foi a determinação dos níveis de ácido benzóico e ácido sórbico em uma variedade de vinhos e sidras brasileiros, de modo a comparar os valores com os máximos permitidos pela legislação. Um total de 49 amostras (sendo 35 vinhos tintos, 11 vinhos brancos e 3 sidras), disponíveis comercialmente, foram analisadas por CLAE com detector de arranjo de diodos. Apesar do uso de ácido benzóico em vinhos e sidras não ser permitido, esse conservador foi detectado em 3 amostras: 1 vinho e 2 sidras em níveis de 295,6, 424,7 e 608,4 mg. $\mathrm{L}^{-1}$, respectivamente. O ácido sórbico foi detectado em $49 \%$ das amostras analisadas com níveis variando de 91,0 a 309,5 mg. $\mathrm{L}^{-1}$. Considerando apenas as amostras nas quais o ácido sórbico foi detectado, o valor médio encontrado foi de 171,2 mg.. ${ }^{-1}$. Em seis amostras de vinho tinto os níveis de ácido sórbico estavam acima do permitido pela legislação brasileira. Os resultados encontrados no presente trabalho mostram que em algumas amostras, os níveis dos ácidos benzóico e sórbico nos vinhos e sidras analisados, assim como a rotulagem desses produtos não estão de acordo com a legislação vigente no Brasil.
\end{abstract}

Palavras-chave: ácido benzóico; ácido sórbico; vinho; CLAE.

\begin{abstract}
This study determined benzoic and sorbic acid contents in Brazilian wines and ciders in order to verify whether these preservatives are used in accordance with Brazilian regulations. Forty-nine samples of commercially available wines (35 red wines, 11 white wines and 3 ciders) were analyzed by HPLC coupled to a photodiode array detector. Although the use of benzoic acid in wines and ciders is not permitted, this preservative was detected in three samples, one wine and two ciders, which contained benzoic acid levels of 295.6, 424.7 and 608.4 mg.. $\mathrm{L}^{-1}$, respectively. Sorbic acid was detected in $49 \%$ of the analyzed samples, with levels ranging from 91.0 to 309.5 mg. $\mathrm{L}^{-1}$. Considering only the samples containing sorbic acid, the mean content detected was $171.2 \mathrm{mg} . \mathrm{L}^{-1}$. Six red wine samples presented sorbic acid content above the maximum level allowed by Brazilian regulations. The results showed that, in some cases, the content of benzoic and sorbic acids in wines and ciders, as well as their labels, do not meet current national regulations.

Keywords: benzoic acid; sorbic acid; wine; HPLC.
\end{abstract}

\section{Introdução}

A preservação de alimentos sempre foi de grande importância na vida do homem. Atualmente os conservadores químicos são amplamente utilizados na conservação de alimentos, estando entre os mais utilizados os ácidos benzóico e sórbico e seus sais de sódio, cálcio e potássio. Eles são normalmente utilizados para inibir o crescimento de fungos e leveduras, sendo também eficientes contra uma ampla gama de bactérias. Esses compostos são mais eficazes em alimentos de baixo $\mathrm{pH}$ e praticamente não apresentam ação em alimentos de $\mathrm{pH}$ neutro $9,10,11,15$.

No Brasil, a legislação vigente permite o uso de ácido sórbico como conservador em vinhos e sidras em um limite máximo de 200 mg. $\mathrm{L}^{-1}$ e $500 \mathrm{mg} . \mathrm{L}^{-1}$, respectivamente. A adição de ácido benzóico não é permitida nesses produtos ${ }^{1}$.

Em diferentes países o uso de aditivos alimentares é limitado através de regulamentos específicos que são estabelecidos com base na sua segurança de uso e na sua necessi-

Recebido para publicação em 13/12/2006

Aceito para publicação em 6/6/2007 (002156)

Universidade Estadual de Campinas - UNICAMP, CP 6121, CEP 13083-862

Campinas - SP, Brasil

2 Instituto de Tecnologia de Alimentos - ITAL, Av. Brasil, 2880, CEP 13070-178,

Campinas - SP, Brasil,

E-mail: tfouni@ital.sp.gov.br

*A quem a correspondência deve ser enviada dade tecnológica. O Brasil, assim como outros países, segue as recomendações do JECFA (Comitê Conjunto FAO/OMS de Peritos em Aditivos Alimentares) para o uso seguro de aditivos. De acordo com o JECFA, a segurança de uso de um aditivo pode ser estabelecida em termos de sua ingestão diária aceitável (IDA) que é a quantidade de um aditivo que pode ser consumida diariamente e por toda vida sem risco apreciável à saúde, expressa em mg por kg de peso corpóreo. IDAs de grupo de 0-5 e 0-25 mg. $\mathrm{kg}^{-1}$ de peso corpóreo foram estabelecidas pelo JECFA para o ácido benzóico e seus sais, benzil acetato, benzil álcool e benzaldeído; e para ácido sórbico e seus sais, respectivamente ${ }^{5,20}$.

Vários estudos de estimativa de ingestão têm sido conduzidos de modo a monitorar qualquer possível excesso no uso de sorbatos e benzoatos de forma a assegurar que as IDAs não sejam ultrapassadas ${ }^{8,17,18,19,21,22}$. No Brasil, a ingestão potencial diária de benzoatos e sorbatos foi estimada por TFOUNI e TOLEDO ${ }^{18}$ tomando como base algumas categorias de alimentos. As ingestões de benzoatos e sorbatos para o consumidor médio ficaram abaixo das IDAs, variando de 0,3 a 0,9, e 0,2 a $0,3 \mathrm{mg} \cdot \mathrm{kg}^{-1} \mathrm{de}$ peso corpóreo, respectivamente. Este trabalho, entretanto, não dispunha de dados relativos aos níveis desses conservadores em vinhos. Apesar das ingestões potenciais diárias estarem significativamente abaixo das IDAs, a verificação desses níveis é importante uma vez que não existem dados disponíveis na literatura referentes à presença dos ácidos benzóico e sórbico em vinhos e sidras brasileiros. 
Nos últimos anos, políticas públicas têm sido propostas de modo a aumentar o consumo de vinho no país, especialmente de marcas nacionais. Além disso, a produção nacional de vinho apresenta boas perspectivas de crescimento devido a recentes investimentos em novas áreas de vinicultura como, por exemplo, no Vale do São Francisco (PE e BA) $)^{6,12,13}$.

Dessa forma, o objetivo do presente trabalho foi determinar, por cromatografia líquida de alta eficiência (CLAE) com detector de arranjo de diodos, os níveis dos ácidos benzóico e sórbico em uma variedade de vinhos e sidras brasileiros de modo a comparar os níveis encontrados com aqueles estabelecidos pela legislação nacional em vigor.

\section{Material e métodos}

\subsection{Padrões e reagentes}

Na realização das análises foram utilizados acetonitrila grau cromatográfico da J. T Baker, outros reagentes da Merck (grau P. A.) e padrões analíticos de ácido benzóico e ácido sórbico da Sigma Chemical Co. Os padrões foram diluídos em água. A água utilizada foi obtida através de um sistema de purificação Milli-Q (Millipore).

\subsection{Amostras}

Diferentes marcas de vinhos e sidras produzidas no Brasil foram adquiridas em supermercados da cidade de Campinas, SP, nos anos de 2004 e 2005. As 49 amostras coletadas e analisadas em duplicata foram divididas da seguinte maneira: 35 amostras de vinho tinto, 11 amostras de vinho branco e 3 amostras de sidra.

\subsection{Preparo das amostras}

A preparação e a análise das amostras foram as mesmas utilizadas por TFOUNI e TOLEDO ${ }^{17}$ na determinação dos ácidos benzóico e sórbico em refrigerantes. Uma alíquota de $2 \mathrm{~mL}$ da amostra foi diluída com água e filtrada em um filtro de 0,45 $\mu \mathrm{m}$ (Millex HV, Millipore Co.). Todas as amostras foram analisadas em duplicata.

\subsection{CLAE}

Na determinação analítica dos ácidos benzóico e sórbico por cromatografia líquida de alta eficiência foi utilizado um sistema cromatográfico Waters composto de bomba quaternária 600, degaseificador em linha, injetor automático 717 e detector de arranjo de diodos 996. Os dados foram adquiridos e processados através do software Milenium 32. Para a separação dos compostos foi utilizada uma coluna C18 (Nova-Pak, $30 \mathrm{~cm} \times 3,9 \mathrm{~mm}$ di, partículas de $4 \mu \mathrm{m}$ ) e fase móvel a uma vazão de $1 \mathrm{~mL} / \mathrm{min}$ composta por $81 \%$ de água, $17 \%$ de acetonitrila e $2 \%$ de acetato de amônio $0,005 \mathrm{M}$ com pH 4,2 ajustado com ácido acético glacial. O volume de injeção foi de $20 \mu \mathrm{L}$. A detecção dos ácidos benzóico e sórbico foi feita nos comprimentos de onda de máxima absorção dos compostos, 228 e 260 nm, respectivamente. Os picos dos compostos nas amostras foram identificados através da comparação dos tempos de retenção com aqueles dos padrões. A identificação e pureza dos picos foram confirmadas através da comparação de seus espectros UV (200 a $400 \mathrm{~nm}$ ) com aqueles dos padrões.

\subsection{Quantificação}

As áreas dos picos nos cromatogramas das amostras de vinho e sidra foram comparadas com as áreas obtidas de soluções aquosas dos padrões puros dos ácidos benzóico e sórbico. Para tanto, foram utilizadas curvas de calibração externa em sete níveis de concentração para a quantificação dos compostos, sendo que cada padrão foi injetado duas vezes no sistema cromatográfico. A concentração dos padrões variou de 0,2 a $10,0 \mu \mathrm{g} \cdot \mathrm{mL}^{-1}$.

O limite de detecção, expresso em $\mu$ g. $\mathrm{mL}^{-1}$, foi determinado utilizando dados da regressão da curva de calibração montada para quantificação dos compostos de interesse, conforme descrito por MILLER e MILLER ${ }^{14}$.

\subsection{Recuperação}

Os testes de recuperação foram feitos adicionando-se quantidades conhecidas dos ácidos benzóico e sórbico em amostras de vinho tinto, em três diferentes concentrações variando de 0,2 a 0,8 mg. $\mathrm{mL}^{-1}$ (ácido benzóico) e 0,05 a 0,2 mg. $\mathrm{mL}^{-1}$ (ácido sórbico). As análises das amostras fortificadas, assim como dos controles foram feitas em duplicata. As recuperações foram calculadas pela diferença entre a concentração dos compostos nas amostras fortificadas e não-fortificadas.

A repetibilidade do método foi avaliada através do coeficiente de variação associado às medidas de cada conservador durante os testes de recuperação.

\section{Resultados e discussão}

Os limites de detecção para os ácidos benzóico e sórbico estão apresentados na Tabela 1. Amostras fortificadas, em 3 diferentes concentrações, apresentaram recuperações médias para cada conservador maiores que $99 \%$. Os resultados obtidos através dos testes de recuperação e seus respectivos coeficientes de variação estão listados na Tabela 1 . Os resultados obtidos estão condizentes com a literatura, que sugere para estudos de recuperação CV inferior a 15\% para análise de compostos em níveis de mg. $\mathrm{L}^{-1} 7$.

Tabela 1. Limite de detecção, recuperação média (R) e coeficiente de variação $(\mathrm{CV})$ dos ácidos benzóico e sórbico em amostras fortificadas de vinho tinto.

\begin{tabular}{lccc}
\hline \multicolumn{1}{c}{ Conservador } & $\mathrm{R}(\%)^{\mathrm{a}}$ & $\mathrm{CV}(\%)$ & Limite de detecção $\left(\mu \mathrm{g} \cdot \mathrm{mL}^{-1}\right)$ \\
\hline Ácido benzóico & 101,2 & 2,6 & 0,06 \\
Ácido sórbico & 99,3 & 5,0 & 0,05 \\
\hline ádia de 3 diferentes concentrações. &
\end{tabular}

Conforme pode ser observado nos cromatogramas obtidos para amostras de vinho tinto e sidra (Figuras 1 e 2), respectivamente, os picos dos ácidos benzóico e sórbico apresentaram adequada separação e resolução. 


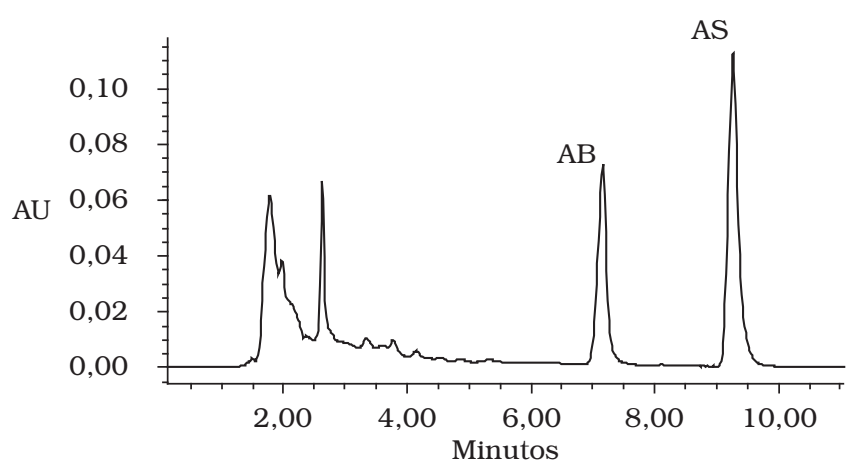

Figura 1. Cromatograma por CLAE referente a uma amostra de vinho tinto. Coluna C18; fase móvel água-acetonitrila-acetato de amônio $0,005 \mathrm{M}$ com pH 4,2 ajustado com ácido acético glacial (81:17:2, v/v/v); vazão: $1 \mathrm{~mL} / \mathrm{min}$; volume injetado: $20 \mu \mathrm{L}$. Detecção a $260 \mathrm{~nm}$. AB: ácido benzóico, AS: ácido sórbico.

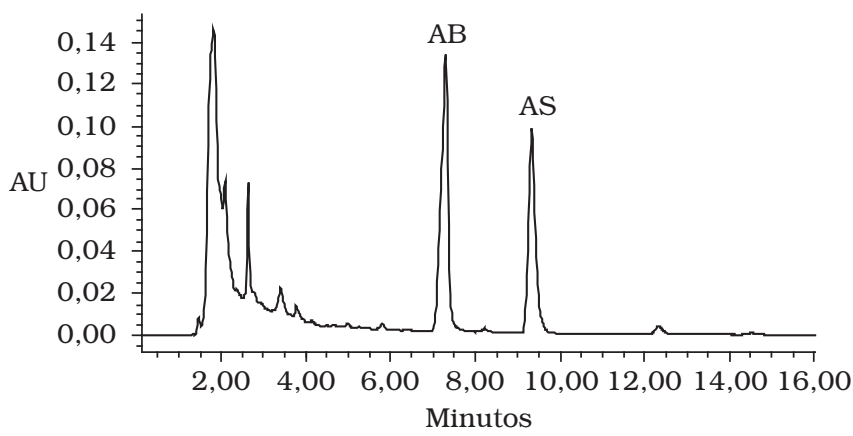

Figura 2. Cromatograma por CLAE referente a uma amostra de sidra. Coluna C18; fase móvel água-acetonitrila-acetato de amônio 0,005 M com pH 4,2 ajustado com ácido acético glacial (81:17:2, v/v/v); vazão: $1 \mathrm{~mL} / \mathrm{min}$; volume injetado: $20 \mu \mathrm{L}$. Detecção a $260 \mathrm{~nm}$. AB: ácido benzóico, AS: ácido sórbico.

A Tabela 2 apresenta os níveis médios de ácido sórbico encontrados nas amostras analisadas. O ácido sórbico foi detectado em $49 \%$ das amostras analisadas com níveis variando de 91,0 a 309,5 mg.L-1 . Através desses resultados pode-se verificar que há grande variação nos níveis de ácido sórbico entre amostras de uma mesma categoria (30\% entre os vinhos tintos) e também entre as amostras de diferentes categorias (até 340\%). Considerando apenas as 25 amostras onde o ácido sórbico foi detectado, o teor médio encontrado foi de 171,2 mg. $\mathrm{L}^{-1}$.

Tabela 2. Média e faixa de concentração de ácido sórbico em amostras de vinhos e sidras.

\begin{tabular}{lccccc}
\hline \multicolumn{1}{c}{ Amostras } & $\mathrm{n}^{1}$ & $\mathrm{n}^{2}$ & \multicolumn{3}{c}{ Ácido sórbico } \\
\cline { 3 - 6 } & & & Faixa $\left(\mathrm{mg} \cdot \mathrm{L}^{-1}\right)^{*}$ & Média $\left(\mathrm{mg} \cdot \mathrm{L}^{-1}\right)^{*}$ & $\mathrm{CV}(\%)$ \\
\hline Vinho tinto & 35 & 15 & $122,1-309,5$ & 188,1 & 30,0 \\
Vinho branco & 11 & 7 & $91,0-192,7$ & 141,7 & 27,0 \\
Sidras & 3 & 2 & $159,9-173,8$ & 166,9 & 5,9 \\
\hline $\mathrm{n}^{1}$ número de amostras analisadas; $\mathrm{n}^{2}$ número de amostras onde o ácido sórbico foi detectado; \\
$\mathrm{e}^{*}$ *alores de média e faixa se referem a amostras onde o ácido sórbico foi detectado.
\end{tabular}

Entre as 35 amostras de vinho tinto analisadas, seis delas (ou seja, 17\%) apresentaram concentrações de ácido sórbico acima do limite máximo permitido de $200 \mathrm{mg} . \mathrm{L}^{-1}$, com níveis variando de 207,8 a 309,5 mg.L L $^{-1}$ (Figura 3).

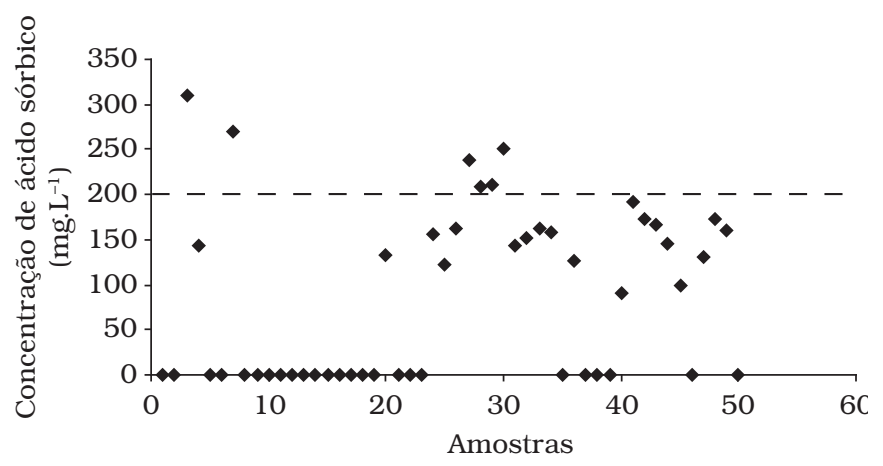

Figura 3. Gráfico de dispersão dos níveis de ácido sórbico. A linha tracejada corresponde ao nível máximo permitido pela legislação (200 mg. $\mathrm{L}^{-1}$ para vinhos).

Em geral, as concentrações de ácido sórbico encontradas nas amostras de vinho tinto apresentaram valores maiores do que aquelas encontradas nas amostras de vinho branco e sidra. A ampla faixa de concentração de ácido sórbico encontrada nas diferentes amostras de vinho e sidra analisadas pode indicar uma adição desse conservador de forma não controlada. Além disso, ao comparar os dados analíticos obtidos com as informações declaradas nos rótulos dos produtos, verificou-se que o ácido sórbico foi detectado em 2 produtos cujos rótulos não mencionavam a sua presença. Esses resultados estão em desacordo com a legislação brasileira, que estabelece que a presença de aditivos alimentares em alimentos e bebidas onde seu uso é permitido deve ser declarada nos respectivos rótulos ${ }^{1,2}$.

Apesar de no Brasil, assim como em outros países, o uso de ácido benzóico em vinhos e sidras não ser permitido ${ }^{1,4}$, esse conservador foi detectado em 3 amostras analisadas: 1 de vinho tinto, e 2 de sidra (Tabela 3).

Tabela 3. Concentração de ácido benzóico nas amostras de vinhos e sidras onde o composto foi detectado.

\begin{tabular}{lc}
\hline Amostra & Ácido benzóico $\left(\mathrm{mg} \cdot \mathrm{L}^{-1}\right)\left(\mathrm{DP}^{*}\right)$ \\
\hline Vinho tinto 8 & $295,6(2,2)$ \\
Sidra 40 & $424,7(0,8)$ \\
Sidra 46 & $608,4(0,1)$ \\
\hline${ }^{*}$ Desvio padrão. &
\end{tabular}

Os resultados obtidos no presente estudo vêm contra o crescente interesse em manter os produtos brasileiros dentro de parâmetros de comercialização tanto em âmbito nacional como internacional. De acordo com as diretivas 95/2/EC ${ }^{3}$ e 98/72/CE ${ }^{4}$, a União Européia estabelece o limite máximo de $200 \mathrm{mg} . \mathrm{L}^{-1}$ de ácido sórbico em vinhos e sidras e não autoriza o uso de ácido benzóico nesses produtos. 


\section{Conclusões}

Os resultados encontrados no presente trabalho indicam que, em algumas amostras, os níveis dos ácidos benzóico e sórbico nos vinhos e sidras analisados, assim como a rotulagem desses produtos, não estão de acordo com a legislação vigente no Brasil.

Uma certa preocupação relacionada ao uso indiscriminado de benzoatos e sorbatos em vinhos e sidras foi levantada. Apesar de ser estabelecido que ingestóes acima da IDA não apresentam, necessariamente, um risco à saúde humana ${ }^{16} \mathrm{e}$ que as ingestões potenciais diárias desses conservadores estão significativamente abaixo das IDAs ${ }^{18}$, o consumo dessas bebidas, no caso de grandes consumidores, pode representar uma importante fonte na ingestão desses conservadores.

Dessa forma, os produtores devem reavaliar o uso de benzoatos e sorbatos em vinhos e sidras de forma a evitar excessos e garantir a segurança dos consumidores e o cumprimento da legislação nacional em vigor.

\section{Agradecimentos}

Os autores gostariam de agradecer ao CNPq pelo apoio ao projeto.

\section{Referências bibliográficas}

1. BRASIL. Resolução CNS/MS n. 04, de 24 de novembro de 1988. Diário Oficial da União, 19 dez. 1988.

2. __. Resolução RDC RDC n. 259, de 20 de setembro de 2002. Diário Oficial da União, 23 set. 2002.

3. EUROPEAN COMISSION. 1995. European Parliament and Council Directive No 95/2/EC of 20 February 1995 on food additives other than colours and sweeteners.

4. 1998. European Parliament and Council Directive $\mathrm{N}^{\circ}$ 98/72/EC of 15 October 1998 on food additives other than colours and sweeteners.

5. FAO/WHO. Summary of evaluations performed by the joint FAO/ WHO expert committee on food additives (JECFA). Washington: ILSI Press. 1999.

6. GUERRA, C. C. et al. Uva para processamento - pós-colheita, $1^{a}$ Edição. Brasília: Embrapa Uva e Vinho, 2003.

7. HORWITZ, W.; KAMPS, L. R.; BOYER, K. W. Quality Assurance in the analyses of foods for trace constituents. Journal of the Association of Analytical Chemistry, v. 63, n. 6, p. 1344-1354, 1980 .
8. ISHIWATA, H. et al. Estimation of preservatives concentrations in food and their daily intake based on official inspection results in Japan fiscal year 1996. Journal of the Food Hygienic Society of Japan, v. 40, n. 3, p. 246-258, 1999.

9. JAY, J. M. Modern food microbiology. New York: Chapman \& Hall, 1996.

10. KIMBLE, C. H. Chemical food preservatives. In: BLOCK, S. S., Desinfection, sterilization and preservation. Philadelphia: Lea \& Febiger, 1977. p. 834-858

11. LÜCK, E. Conservación química de los alimentos. Zaragoza: Editorial Acribia, 1977.

12. MELlo, L. M. R. Embrapa Uva e Vinho. Atuação do Brasil no mercado internacional de uvas e vinhos - panorama 2004. [internet]. Disponível em <http://www.cnpuv.embrapa.br $>$. Acesso em: 21 out. 2004.

13. _. Embrapa Uva e Vinho. Produção e comercialização de uvas e vinhos - panorama 2005. [internet]. Disponível em $<$ http://www.cnpuv.embrapa.br>. Acesso em: 23 mar. 2006.

14. MILLER, J. C.; MILLER, J. N. Statistics for analytical chemistry. Chichester: Ellis Horwood Limited, 1993.

15. SOFOS, J. N. Antimicrobial agents. In: MAGA, J. A. e TU, A. T., Food additive toxicology. New York: Marcel Dekker Inc, 1995. p. 501-529.

16. SPEIJERS, G. J. A. Precision of estimates of an ADI (or TDI or PTWI). Regulatory Toxicology and Pharmacology, v. 30, n. 2, p. S87-S93, 1999.

17. TFOUNI, S. A. V.; TOLEDO, M. C. F. Determination of benzoic and sorbic acids in Brazilian food. Food Control, v. 13, n. 2 , p. 117-123, 2002.

18. Estimates of the mean per capita daily intake of benzoic and sorbic acids in Brazil. Food Additives and Contaminants. v. 19, n. 7, p. 647-654, 2002.

19. VERGER, P. et al. Estimation of the distribution of the maximum theoretical intake for ten additives France. Food Additives and Contaminants, v. 15, n. 7, p. 759-766, 1998.

20. WHO. Evaluation of some food additives and contaminants. Technical Report Series, n. 868, 1997.

21. Evaluation of certain food additives (Fifty-first Report of the Joint FAO/WHO Expert Committee on Food Additives). WHO Technical Report Series, n. 891, 2000.

22. YOON, H. J. et al. Assessment of estimated daily intakes of benzoates for average and high consumers in Korea. Food Additives \& Contaminants, v. 20, n. 2, p. 127-135, 2003. 